\title{
Prognostic factors correlation between the cell cycle phases and apoptosis in basal cell carcinoma
}

\author{
Cyro Festa-Neto ${ }^{1}$, Durvanei Augusto Maria ${ }^{2}$ \\ ${ }^{1}$ Oncology and Surgery Divisions of the Department of Dermatology of the Sao Paulo, University School of Medicine, Sao Paulo, \\ Brazil; \\ ${ }^{2}$ Biochemistry and Biophysical Laboratories, Butantan Institute, Sao Paulo, Brazil. \\ Email: durvanei@usp.br
}

Received 8 July 2011; revised 15 August 2011; accepted 12 October 2011.

\begin{abstract}
Basal cell carcinoma (BCC) is the most common skin malignancy, are found in various forms depending on their clinical and biological behavior. The objective of study was analyzed the phases of the cell cycle and correlations between BCC of low and high risk of recurrence and correlation prognostic factors. The quantity of content DNA in tissues of normal skin, showed small amount of cells in apoptosis and mostly in phase quiescent and rare aneuploidy cells. In BCC, apoptosis was higher in the BCC at high risk than low risk, probably due to their high rates of cell proliferation, and present of aneuploidy cells, when compared to the average percentage of aneuploidy. The DNA content from cells of normal skin shows that the majority is in the quiescent phase; compatible with tissues that are is refreshing. There is presence of apoptosis in the epidermis by probable normal process of differentiation. The aneuploidy in BCC showed a direct correlation with the degree of tumor aggressiveness.
\end{abstract}

Keywords: Basal Cell Carcinoma; Cell Cycle Phases; Apoptosis; Nonmelanoma Skin Cancer; Aneuploidy

\section{INTRODUCTION}

Basal cell carcinoma (BCC) is the commonest type of malignancy acquired during human lives [1]. These tumors are found in various forms depending on their clinical and biological behavior. The majority has low aggressiveness with only local invasion and destruction, but some are aggressive with deep infiltration, multiple recurrences, often leading aesthetic and functional deformities [2]. Therefore it is important to properly classify the BCC according to their histological pattern, helping the dermatologist to estimate those with greater chance of recurrence and those require aggressive treatment and close follow-up. The characteristics of solid tumors, that is, uncontrolled proliferation, derangement of cellular and morphological differentiation, invasion, and colonization to distant organs, can be attributed, in part, to alterations in communications between neoplastic cells and the normal cells in their immediate microenvironment. Thus, skin cells within or surrounding the cancerous tissue are not idle bystanders, but rather active participants whose function in shaping the aggressive features of the neoplasm depends on the structural and functional aspects of the interactions [3]. Unfortunately, morphological methods are not always reliable and sometimes low risk tumors may have an aggressive behavior while some high risk has a benign course. Several reports tried to correlate the type of BCC cells with clinical tumor behavior using other methods such as morphometry and immunohystochemistry. Although they could be used as new markers of tumor aggressiveness, high costs and practical difficulties may represent obstacles to its routine application. Changes in DNA content occur frequently in tumors and are a reflection of the overall genetic changes, chromosome and sub chromosome, which play a crucial role in development and tumor progression [4]. This parameter is considered to be an important biological and prognostic variable in human cancer [5]. Due to the close association between chromosomal changes in human cells and the occurrence of cancer, the study of molecular genetics, cytogenetic and cellular cycles have been emphasized. In this study, we analyzed the phases of the cell cycle, apoptosis and debris/necrosis by flow cytometry and investigated the possible differences between basal cell carcinomas of low and high risk of recurrence.

\section{PATIENTS}

Seventy-five tumors from outside patients from the Oncology and Surgery Divisions of the Department of Dermatology of the Sao Paulo University School of Medi- 
cine were studied. Samples from patients with suspected BCC were collected thought 4 - 5 mm punch biopsy. Surgical excision of the tumor was performed in confirmed cases of BCC and/or small lesions. Tumor fragments were divided and placed in formalin and in liquid nitrogen, and stored for further histological examination and flow cytometry analysis, respectively. Histological exams were reviewed by two separate investigators. The Royal College of Pathology criteria [6] were employed in confirmed cases of BCC to identify the degree of risk of recurrence. Tumors were considered as high risk when $50 \%-100 \%$ of the lesion was constituted of superficial, infiltrative, micronodular and morpheic histopathological patterns and/or when they reached the margin of resection. Nodular and nodular-cystic forms and variations were considered low risk tumors. Forty-two BCC samples out of 75 biopsies were selected for the study and 27 normal skin samples from herniorrhaphy and blepharoplasty from healthy volunteers were used as controls.

\section{CELL CYCLE PHASES ANALYSIS BY FLOW CYTOMETRY}

The determination of the cell cycle was performed on FACSCalibur flow cytometer (BD) using the methodology of propidium iodide. Samples of tumors and normal skin were removed from the cryopreservation in liquid nitrogen and incubated for 10 minutes at room temperature with $30 \mathrm{mg} / \mathrm{ml}$ solution of trypsin (Sigma Chemical Co., USA) and, then macerated. Cell suspensions were centrifugated in the presence of $5 \mathrm{mg}$ of trypsin inhibitor (Sigma Chemical Co., USA) and $0.1 \mathrm{mg} / \mathrm{l}$ ribonuclease-A (Sigma Chemical Co.,USA) at $800 \mathrm{~g}$, at $4^{\circ} \mathrm{C}$ for $15 \mathrm{~min}-$ utes. The cells were incubated with $18 \mathrm{ug} / \mathrm{ml}$ propidium iodide (Sigma Chemical Co., USA) for 15 minutes at room temperature, followed by acquisition and analysis in flow cytometry. The analysis was performed with the identification of the cell population in gate defined homogeneous cell population acquired by the acquisition of Cell Quest Software. The DNA histograms were analyzed by ModFit LT DNA cell cycle analysis software for flow cytometry.

\section{STATISTICAL ANALYSIS}

One-way ANOVA and Bonferroni correction were performed to identify differences among multiple groups. Independent or paired t test was performed to identify differences between two groups.

\section{ETHICAL LEGAL}

This study was approved by the Department of Dermatology, Faculty of Medicine, University of Sao Paulo and the Commission on Ethics in Research of the Clinical
Board of Hospital of the Faculty of Medicine, University of Sao Paulo. Donor's volunteers who formed the normal control group were informed about the research protocol and signed the Term of Free and Informed Consent.

\section{RESULTS}

Analysis of the phases of the cell cycle, apoptosis and DNA content by flow cytometry which results were expressed as mean percentage of cells in different phases of the cell cycle (aneuploidy, G2/M, S, G0/G1, apoptosis) and debris/necrosis cell

The cells of normal skin showed G0/G1 phase of the cell cycle (50.3\%) followed by G2/M phases (3.3\%), S (2.2\%) and apoptosis (0.3\%). Aneuploidy was found in $0.01 \%$ of the cells and $33 \%$ of the samples were cellular debris. The samples of BCC, $14.4 \%$ the cell population in $\mathrm{S}$ phase followed by $\mathrm{G} 0 / \mathrm{G} 1$ phases (11.1\%), apoptosis (7.8\%) and G2/M (6.4\%). Aneuploidy was found in 3.1\% of the cells and $60.5 \%$ of the sample were cellular debris (Figures 1(a) and (b)).

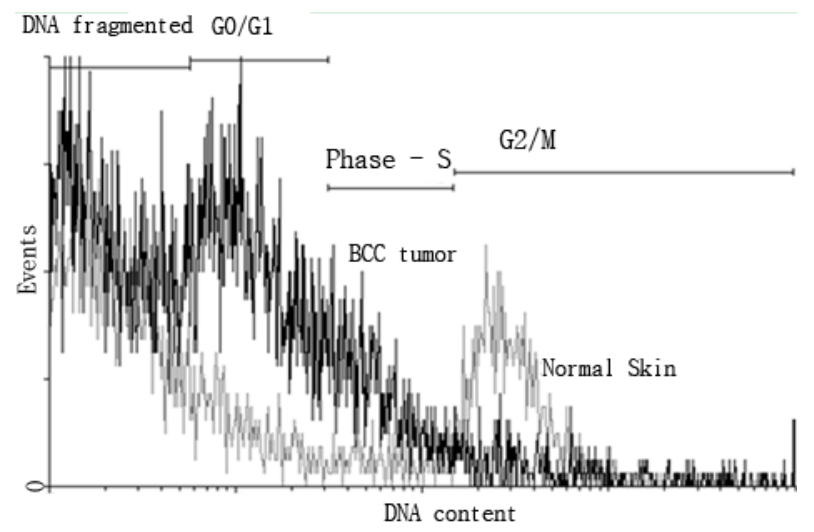

(a)

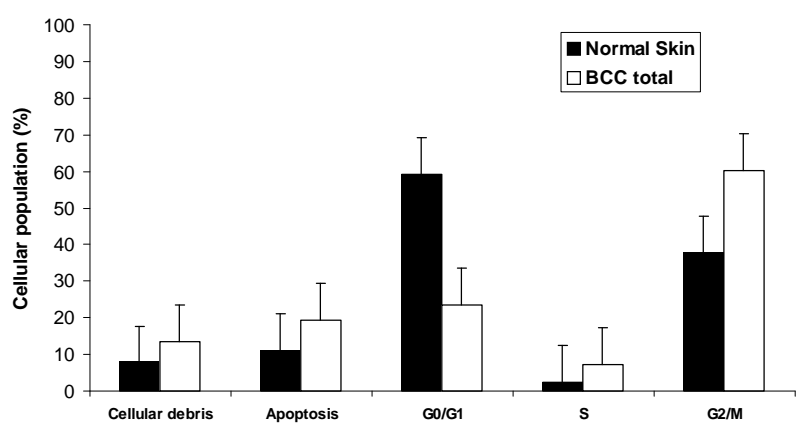

(b)

Figures 1. Histogram of normal cells of the skin (gray) and BCC tumor (black) stained with iodide propidium for DNA content analysis by flow cytometry. a) The quadrants represent analyzed the proportions of cells in G2/M proliferative rate cell division; phase S synthesis; G0/G1 quiescent cell, no proliferative and debris and DNA fragmented. The DNA his- tograms were analyzed by ModFit LT DNA cell cycle analysis software. b) The distribution curves of the content of DNA were compared between normal skin samples and BCC total. 
The low risk BCC, showed increased in S phase population tumor cells (10.9\%), followed by quiescent cells G0/G1 (7.5\%), apoptosis (6.4\%) and G2/M (5.8\%). Aneuploidy was found in $1.9 \%$ of the cells and $70 \%$ of the samples were cellular debris.

The high risk basal cell carcinomas $(n=21)$, showed significantly enhanced the population tumor cells in phases of synthesis (17.8\%), followed by G0/G1 phases (14.5\%), apoptosis (9.1\%) and increased cells in proliferation G2/M (7.0\%). Aneuploidy was found in $4.2 \%$ of the cells and $51.1 \%$ of the sample were cellular debris. Comparative analysis of the phases of the cell cycle, apoptosis and DNA content by flow cytometry, the results of mean percentage in samples of normal skin and basal cell carcinomas, significant differences were demonstrated between the phases S (normal skin-2.3\% and BCC 14.4\%), G2/M (normal skin-3.3\% and BCC 6.4\%), G0/G1 (normal skin-50.3\% and BCC 11.1\%) and apoptosis (normal skin $0.29 \%$ and BCC 7.8\%). Statistical analysis enhanced show the population in changes contends of DNA, aneuploids (normal skin $0.01 \%$ and 3.1\% BCC) and cellular debris/necrosis (normal skin 33.3\% and BCC 60.9\%) (Figure 2).

The samples of BCC compared of high and low risk presented differences significantly were demonstrated in phases S (low risk $10.9 \%$ and high risk 17.8\%), G0/G1 population (low risk $7.5 \%$ and high risk 14.5\%) and apoptosis (low risk BCC 6.4\% and high risk BCC 9.1\%). Statistical differences between in aneuploids (low risk $1.9 \%$ and high risk BCC 4.2\%) and cellular debris/necrosis (low risk 70\% and high risk 51.1\%) (Figures 3 (a) and (b)).

\section{DISCUSSION}

The DNA aneuploid content is one of the most common biological characteristic in human solid tumors and may contribute to tumor formation and often to acquired resistance to some chemotherapeutic agents. The tumor cells become aneuploid as a result of aberrant mitotic divisions caused by previous defects in cytokinesis or centrosome amplification or impaired mitotic checkpoint response. As seen in other human cancers, aneuploidy is a multi-step process in carcinogenesis and correlates with tumor progression [7-9]. Our first goal was the standardization of the controls. Samples of normal skin of patients the tumors studied were more difficult to obtain from the ethical point of view. Additionally, some studies have shown that even normal skin of non-exposed area of carrier of skin cancer may have changes in their cell cycle and the increase in S and G2/M fraction [10, 11]. Few publications that make reference to the study of normal skin by flow cytometry and are related to methods for evaluating the kinetics of epidermal cells. The location and local injure can alter the previous results of the study, but gender, age, time of day, length of storage in liquid nitrogen appear to be less important [10, 12-14]. Renewable tissues have the ability to change their proliferative capacity against a subsequent injury to repair. The skin is the prototype of a tissue in constant renewal. Its cell cycle has duration of 209 hours, the epidermal cells turnover is 326 hours, and the duration of $\mathrm{S}$ phase is 8.3 hours and 1.2 hours of $\mathrm{M}$ phase mitotic. The analysis of DNA from skin cells of normal controls, expressed as mean percentage of cells in different phases

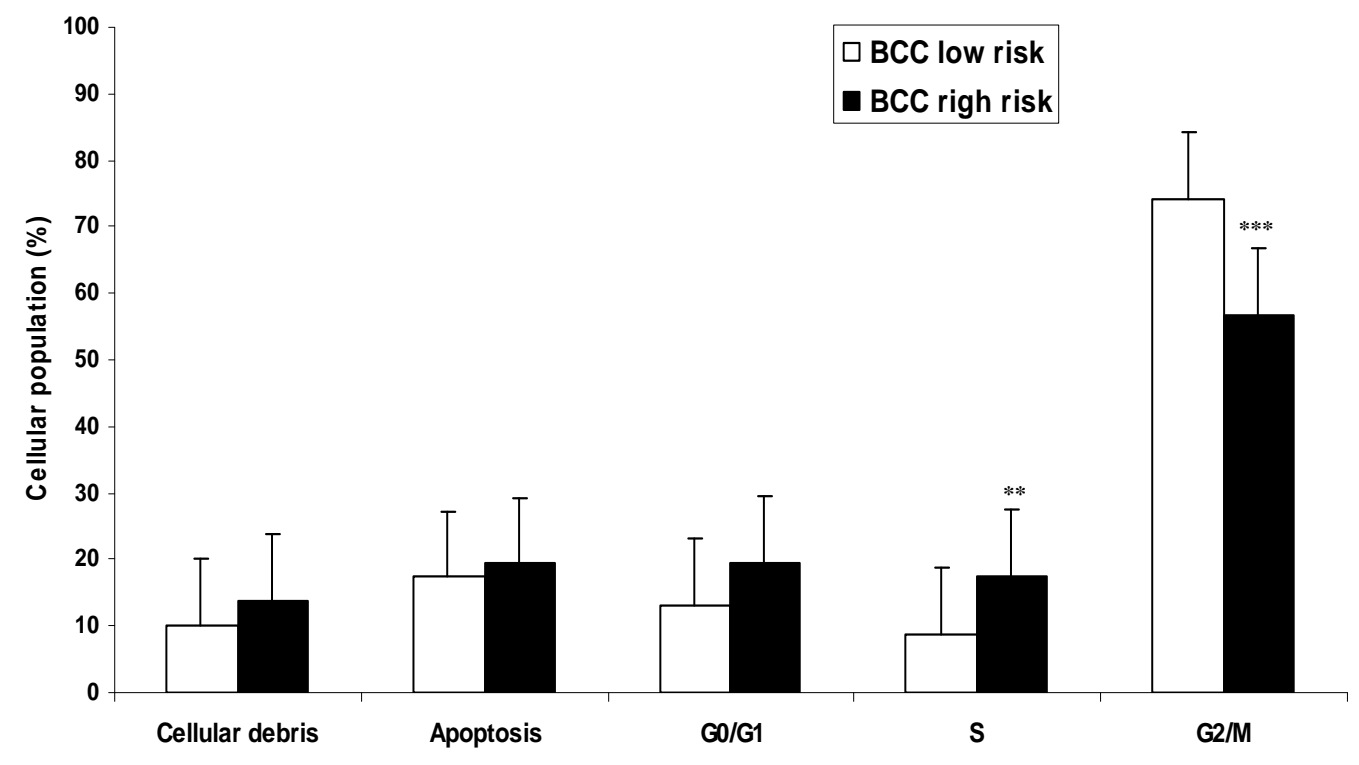

Figure 2. Analysis of DNA from cells of basal cell carcinoma of low and high risk by flow cytometry. Results were expressed as mean percentage of cells in different phases of the cell cycle: G2M, synthesis S, G0/G1, apoptosis and cellular debris. *Significant difference with $\mathrm{P}<0.001$ ANOVA test. 


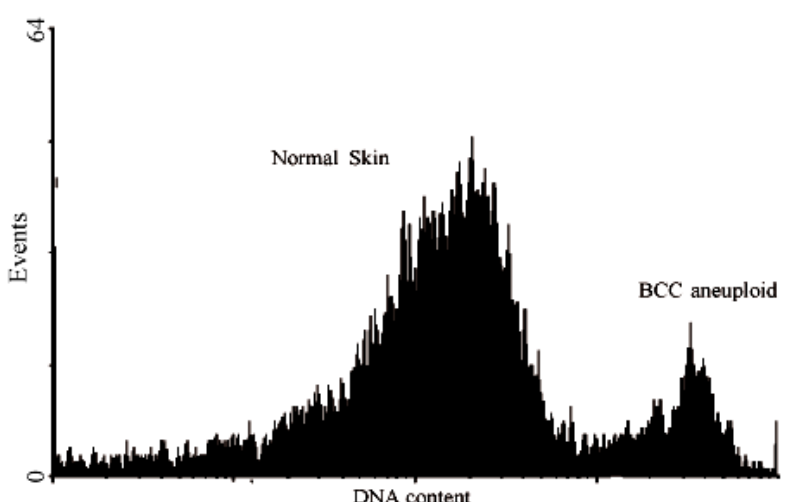

(a)

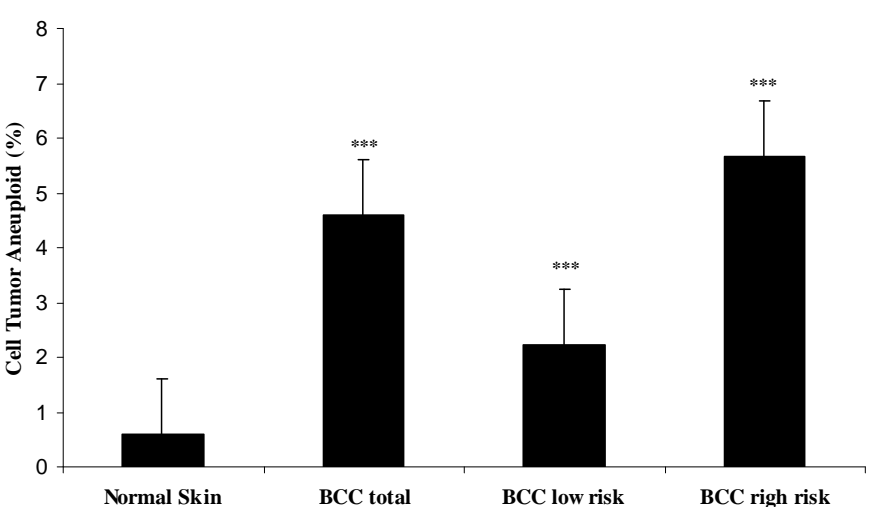

(b)

Figure 3. Histogram showing cell cycle parameters using flow cytometer software showing:normal diploid pattern on normal skin and aneuploid pattern BCC tumor. Analysis of percentage of tumor cells with changes in the quantity of DNA, aneuploidy, BCC of low and high risk, compared to normal skin (a). DNA histogram is defined as DNA diploid when the predominant or G0/G1 peak of the histogram centers in the range of DNA content equal to that of a known diploid cell population and the S-phase and G/M phase content is relatively low (b). In skin normal tissues and most low-grade or slowly proliferating carcinomas, approximately $85 \%$ of the cell population forms the G0 and G1 peak and 15\% of the cells are in the S phase and G2/ M phases. *Significant difference with P > 0.01 ANOVA test.

phase and a small peak in the G2/M phase, this pattern is compatible with tissues that are renewed. The presence of apoptosis in the epidermis is a common phenomenon both in the morphogenesis of the fetal skin and in maintaining the skin in adults. An example is the mechanism of apoptosis activated during the normal process of differentiation of keratinocytes. Apoptotic cells can be found occasionally in normal human keratinocyte differentiation and activation caspase during apoptosis is apparently required for the normal loss of the nucleus [15]. In control groups studies found apoptosis in the frequency of $0.29 \%$. The presence of aneuploidy in normal skin is not expected. The fact that $0.01 \%$ of the cells of the skin can be explained by aneuploidy detection by flow cytometry of cell population in an interface of the separation of daughter cells. Moreover, the presence of aneuploidy cells is characteristic of tumors. Of the 42 BCC studied, 31 samples showed aneuploidy cells. In the BC$\mathrm{C}$, there are many publications with only occasional [13, $16,17]$. The only study with a large number of cases [18] showed average of $19 \%$ of aneuploidy in 509 BCC studied. Unfortunately, this is a service of summons and could not recover it or explain the reason for the signifycant difference with our findings (73.8\% of samples of BCC with cellular aneuploidy). There is evidence of the association of ploidy to prognosis of solid tumors thus diploid DNA indicates less aggressive behavior and better prognosis of the tumor, whereas DNA aneuploidy, more aggressive behavior and worse prognosis [19]. Aneuploidy was seen by Q-banding method in more than half of the cases and was mostly loss of the sex chromosome [20]. Applied to work on skin cancer are few and still controversial. So far, studies exist which relate the possible differences between histology subtypes of BCC with low and high risk of recurrence using the index as a measure of DNA, which is one of the purposes of this research. Comparing the average percentage of aneuploidy in BCC cells of low and high risk and could show differences between them low and high risk; this may be a parameter of prognostic index in the BCC. The development of BCC might be correlated with aneuploidy, which acquired accumulation of genetic mutations [9]. Only two studies tried to analyze the DNA of cells of BCC by flow cytometry and differentiate those according morphological and histological aspects. The studies revealed that, BCC with differential keratotic and metatypical had larger amounts of aneuploidies that the BCC without differentiation keratotic (solid and cystic) [21, 22]. It is known that the fractions of $\mathrm{S}$ and G2/M cell cycle population progressively increased in cells of the area non-exposed to solar radiation, exposed area and tumor tissue [16]. All tumors analyzed in this study showed the average percentage of cells in S phase of cell cycle increased in relation to normal skin. Associated with aneuploidy, the proliferative index, the rate of cells in $S$ phase is another possible indicator of prognosis of the biological aggressiveness of solid tumors [23]. DNA aneuploidy or tetraploidy and histology of well differentiated squamous cell carcinoma were significantly associated with metastasis to regional lymph nodes [24]. The correlation between the fractions of cells in $S$ phase with aggressiveness of the tumors studied showed that among the high risk BCC show the S fraction higher than the low risk of BCC. The analysis of DNA content from skin cells of normal controls shows that the majority is in the G0/G1 phase of the cell cycle, a small percentage in $\mathrm{S}$ 
phase and G2/M phase in standard compatible tissue renewal. Various kinds of tumors in the percentage of cells in $\mathrm{G} 0 / \mathrm{G} 1$ phase was shown to be lower than the controls, total BCC, low and high risk. Among the differences between BCC tumors are low risk and high risk, showing that the greater the cellular in tumors. The G2/M phase represents, together with the $\mathrm{S}$ phase, cells with higher proliferative capacity. In all tumors studied noticed an increase in the percentage of cells in this phase is correlated with increased aggressiveness of the tumor. The apoptosis is related to the physiological regulation of homeostasis in tissues. Most cancer cells exhibit defective apoptotic mechanisms or develop mechanisms to evade apoptosis, allowing them to develop in an uncontrolled way. Thus, restoring apoptosis is a promising way to treat cancers. In fact, some treatments already function by the apoptosis mechanism. All tumors studied showed percentages of cells in apoptosis greater than normal skin. Among the differences between BCC tumors were of high and low risk. Apoptosis is controlled by the regulatory system of growth of tissues in the body. It is modulated by events in the transduction of signals and depends on the activation of the expression of genes in cell death. The proto-oncogene Bcl-2, located on chromosome 18, encodes an inner mitochondrial protein that protects cells from apoptosis. Translocation of this gene leads to over expression of this product, with consequent inhibition of programmed cell death and increased survival of normal and neoplastic cells. The Bcl-2 alone is not sufficient for neoplastic transformation, but the cells that survive the translocation of $\mathrm{Bcl}-2$ are targets for the addition of genetic changes and, eventually, a malignant clone can be produced. Ultraviolet radiation (UVR) is the factor of greatest importance in the development of BCC. The UVR leads to changes in the DNA of cells and these are removed by the process of apoptosis. The lack of regulation of expression of Bcl-2, also induced by UVR, suppresses cell death leading to the accumulation of oncogene mutations and selection of more aggressive cell clones. Probably, in oncogenesis, the mutation of proto-oncogene Bcl-2 leads to inhibition of apoptosis and increased survival of normal and neoplastic cells. Later, once the tumor developed, their proliferation leads to an attempt to programmed cell death increased to contain its expansion. Previous studies have shown that BCC cells express Bcl-2 and p53, more intensely, the less aggressive [25-30]. Therefore, the higher the aggressiveness of the BCC, lower expression of Bcl-2 and, consequently, greater apoptosis as observed in our cases. The study of apoptosis of tumor cells of BCC of nonaggressive behavior and aggressive by other methods showed also that non-aggressive BCC have lower rates of apoptosis, that aggressive which is a parameter that can give the poor prognosis tumor. High-risk BCCs are characterized by one or more of the following: an increased probability of subclinical extension, incomplete excision, aggressive local invasive behavior, and local recurrence [31]. Tumor microenvironment refers to the unique properties of tissue microenvironment conferred by abnormal interactions between tumor and host cells. In normal skin, cellular debris are represented by amorphous materials such as keratin, collagen, elastin, reticulin and cellular components as dead keratinocytes, endothelial cells, inflammatory and others. In tumors, cellular debris is made of tumor stroma matrix and tumor cells killed by cell degeneration or necrosis. The percentage of total debris and dead cells with DNA fragmented is lower in normal skin than in tumors, perhaps due to the fact that there is increased proliferation in tumors, most cellular components and, consequently, a greater proportion of cell death. Among the tumors noted that there are differences. The reduced amount of cellular debris observed between the various types is associated with greater severity. It was not possible to compare the size of tumors or time of evolution with the amount of cellular debris.

In summary, the analysis of DNA from cells of normal skin shows that the majority is in the G0/G1 phase, a small percentage in $S$ phase and G2/M phase in the small peak, standard, compatible with tissues that are is refreshing. Intrinsic biological factors and extrinsic management factors play a role in the development and progression of aggressive BCC. DNA ploidy and cell cycle has been simultaneously assessed in a large series of skin tumors to explore the prognostic value of DNA ploidy status and direct correlation with the degree of tumor aggressiveness.

\section{REFERENCES}

[1] Miller, S.J. (1995) Etiology and pathogenesis of basal cell carcinoma. Clinics in Dermatology, 13, 527-536. doi:10.1016/0738-081X(95)00062-K

[2] Dixon, A.Y., Lee, S.H. and MacGregor, D.H. (1989) Factors predictive of recurrence of basal cell carcinomas. American Journal of Dermatopathology, 11, 222-232. doi:10.1097/00000372-198906000-00005

[3] Pietras, K. and Ostman, A. (2010) Hallmarks of cancer: interactions with the tumor stroma. Experimental Cell Research, 316 ,1324-1331. doi:10.1016/j.yexcr.2010.02.045

[4] Cornelisse, C.J., Deville, P. and Smit, V.H. (1993) DNA Content as a genetic marker of cancer cells. In: Bauer, K.D., Duque, R.E. and Shankey, T.V., Eds., Clinical Flow Cytometry: Principles and Application, Williams \& Wilkins; Baltimore, 3-12.

[5] Barlogie, B. Johnston, D.A., Smallwood, L. et al. (1982) Prognostic implications of ploidy and proliferative activ- 
ity in human solid tumours. Cancer Genetics and Cytogenetics, 6, 17-28. doi:10.1016/0165-4608(82)90017-6

[6] Slater, D.N. (2004) Standards and minimum datasets for reporting cancers minimum dataset for the histopathological reporting of common skin cancers. Royal College of Pathologists, London, 1-23.

[7] Duesberg, R., Li, P., Fabarius, A., Hehlmann, R. (2005) The chromosomal basis of cancer. Cellular Oncology, 27, 293-318.

[8] Kops, G.L., Weaver, B.A., Cleveland, D.W. (2005) On the road to cancer: Aneuploidy and the mitotic checkpoint. Nature Reviews Cancer, 5, 773-785. doi:10.1038/nrc1714

[9] Koutsami, M.K., Tsantoulis, P.K., Kouloukoussa, M., et al. (2006) Centrosome abnormalities are frequently observed in non-small-cell lung cancer and are associated with aneuploidy and cyclin E overexpression. Journal of Pathology, 209, 512-521. doi:10.1002/path.2005

[10] Frentz, G., Moller, U., Christensen I. (1980) DNA flow cytometry of human epidermis: I. methodological studies on normal skin. Journal of Investigative Dermatology, 74, 119-21. doi:10.1111/1523-1747.ep12535006

[11] Frentz, G. and Moller, U. (1981) DNA measurements by single nuclei flow cytometry in human actinic skin lesions. Journal of Investigative Dermatology, 77, 3133. doi:10.1111/1523-1747.ep12495660

[12] Frentz, G., Moller, U. and Keiding, N. (1982) DNA flow cytometry of human epidermis. The effect of serial biopsy sampling at various times. British Journal of Dermatology, 107, 7-14. doi:10.1111/j.1365-2133.1982.tb00283.x

[13] Frentz, G. and Moller, U. (1983) DNA flow cytometry of human epidermis: Interindividual and regional variations in normal skin. Clinical and Experimental Dermatology, 1, 19-26. doi:10.1111/j.1365-2230.1983.tb01739.x

[14] Gray, J.W., Carver, J.H., George, Y.S. and Mendelsohn, M.L. (1977) Rapid cell cycle analysis by measurement of the radioactivity per cell in narrow window in $\mathrm{S}$ phase. Cell and Tissue Kinetics, 10, 97-109.

[15] Weil, M., Raff, M.C. and Braga, V.M. (1999) Caspase activation in the terminal differentiation of human epidermal keratinocytes. Current Biology, 8, 361-364. doi:10.1016/S0960-9822(99)80162-6

[16] Frentz, G. and Moller, U. (1984) DNA flow cytometry of the epidermis of patients with multiple epidermal carcinomas. British Journal of Dermatology, 111, 271-277. doi:10.1111/j.1365-2133.1984.tb04723.x

[17] Frentz, G., Møller, U. and Larsen, J.K. (1985) DNA flow cytometry of human epidermal tumours. Intra- and intertumour variability in ploidy and proliferative characteristics. Virchows Arch B Cell Pathol Incl Mol Pathol., 48, 175-183. doi:10.1007/BF02890126

[18] Buchner, T.H., Hiddemann, W. and Schumann, J. (1985) DNA aneuploidy a common cell marker in human malignancies and its correlation to grade, stage, and prognosis. In: Buchner, T.H., Bloomfield, C.D., Hiddemann, W., Hassfeld, D.K. and Schumann, J., Eds., Tumor Aneuploi$d y$, Springer-Verlag, New York, 41-52.
[19] Friedlander, M.L., Hedley, D.W. and Taylor, I.W. (1984) Clinical and biological significance of aneuploidy in human tumours. Journal of Clinical Pathology, 37, 961974. doi:10.1136/jcp.37.9.961

[20] Koyama ,N., Nishihira, J., Nakabayashi, H., et al. (2000) Aneuploidy of sex chromosomes in basal cell carcinoma: its clonality and involvement in the development of carcinogenesis. International Journal of Oncology, 16, 5-23.

[21] Fortier-Beaulieu, M., Laquerrière, A., Thomine, E., Lauret, P. and Hémet, J. (1994) NA flow-cytometric analysis of basal cell carcinomas and its relevance to their morphological differentiation: A retrospective study. Dermatology, 188, 94-99. doi:10.1159/000247109

[22] Miller, S.J. (1991) Biology of basal cell carcinoma. Journal of the American Academy, 24, 1-13. doi:10.1016/0190-9622(91)70001-I

[23] Pappas, A.A., Maners, A.W., Owens, R.B., Thompson, J.R., King, J.C. and Dinehart, S.M. (1992) Deoxyribonucleic acid (DNA) ploidy and proliferative characteristics of metastatic squamous cell carcinoma determined by flow cytometric analysis. Journal of Dermatologic Surgery and Oncology, 18, 957-960.

[24] Robinson, J.K., Rademaker, A.W., Goolsby, C., Traczyk, T.N. and Zoladz, C. (1996) DNA ploidy in nonmelanoma skin cancer. Cancer, 77, 284-291. doi:10.1002/(SICI)1097-0142(19960115)77:2<284::AID -CNCR10>3.0.CO;2-Y

[25] Bolshakov, S., Walker, C.M., Strom, S.S., et al. (2003) p53 mutations in human aggressive and nonagressive basal and squamous cell carcinomas. Clinical Cancer Research, 9, 228-234.

[26] Cerroni, L. and Kerl, H. (1994) Aberrant bcl-2 protein expression provides a possible mechanism of neoplastic cell growth in cutaneous basal-cell carcinoma. Cutaneous Pathology, 21, 398-403. doi:10.1111/j.1600-0560.1994.tb00279.x

[27] Delehedde, M., Cho, S.H., Sarkiss, M., et al. (1999) Altered expression of bcl-2 family member proteins in nonmelanoma skin cancer. Cancer, 85, 1514-1522.

[28] Staibano, S., Lo Muzio, L., Pannone, G., et al. (2001) Interaction between bcl-2 and p53 in neoplastic progression of basal cell carcinoma of the head and neck. Anticancer Research, 21, 3757-3764.

[29] Wikonkal, N.M., Berg, R.J.W., van Haselen, C.W., et al. (1997) Bcl-2 versus p53 protein expression and apoptotic rate in human nonmelanoma skin cancer. Archives of Dermatology, 133, 599-602. doi:10.1001/archderm.1997.03890410055007

[30] Tille, C.M.L.J., Stavast-Kooy, A.J.W., Remaekers, F.C.S. and Neumann, H.A. (2002) Bax expression and growth behaviour of basal cell carcinomas. Journal of Cutaneous Pathology, 29, 79-87. doi:10.1034/j.1600-0560.2002.290203.x

[31] Staibano, S., Muzio, L.L., Mezza, E., Argenziano, G., Tornillo, L., Pannone, G. and De Rosa, G.(1999) Prognostic value of apoptotic index in cutaneous basal cell carcinomas of head and neck. Oral Oncology, 35, 541- 547. doi:10.1016/S1368-8375(99)00028-7 